\title{
A pigeon fancier's lung; subacute on chronic hypersensitivity pneumonitis
}

\author{
Peiris A
}

\begin{abstract}
:
Pigeon fancier's lung, a form of hypersensitivity pneumonitis (HP), is a rare but important occupational and recreational preventable cause of severe and debilitating breathlessness. We report a case of a 44-year-old Sri Lankan lady presenting with progressive severe breathlessness due to pigeon fancier's lung. She has bred pigeons for 20 years. She was hypoxemia at rest. There were reticular nodular opacities and diffuse ground glass opacities, interand intralobular thickening with subpleural sparing on high resolution imaging. She had marked improvement to glucocorticoid pulses. We diagnosed this patient as subacute on chronic HP considering clinical and radiological evidence. Avoidance of allergen and steroids advised with regular follow up to monitor progression.
\end{abstract}

\section{Introduction:}

Hypersensitivity pneumonia (HP), or extrinsic allergic alveolitis, is a spectrum of interstitial, alveolar and bronchiolar lung disease caused by non IgE mediated immunologic reaction occurring within lung parenchyma due to sensitization and subsequent recurrent exposure to various inhaled antigens.This may lead to irreversible damage. Pigeon fancier's lung, a form of hypersensitivity pneumonitis, is an occupational and recreational preventable cause of HP. It is caused by exposure to air-borne avian antigen which provokes a hypersensitivity reaction in a susceptible host.We report a case of a 44-year-old Sri Lankan lady suffering with severe breathlessness due to pigeon fancier's lung. She has bred pigeons for 20 years.

\section{Case presentation:}

A 44-year-old non-smoking housewife from an urban area admitted to the emergency treatment unit of National Hospital due to progressive dyspnea at rest for six days. She had an exertional dyspnea of Modified Medical Research Council(mMRC) grade 2 severity, a dry cough and fatigue for past 3 months.

Postgraduate Traine in Clinical Medicine

Corresponding Author:

Anushka Peiris

peiris.anushka88@gmail.com

(iD https://orcid.org/0000-0002-2894-1828
Recently she has developed acute worsening of dypnoeaup tomMRC grade 4 associated with fever and productive cough for six days. She had neither pleuritic type chest pain nor hemoptysis. She had no childhood asthma. She denied any oral ulcers, photosensitivity rashes, epistaxis, arthralgia and myalgia. There were no known allergies. She had not been on any long-term medications. She had no self or family history of any rheumatological diseases. She has been exposed to pigeon dust since her marriage due to pigeon keeping and cleaning the lofts at home for twenty years.

On examination, she was breathless at rest but alert and rational. She was afebrile. She had no cervical lymphadenopathy, finger clubbing or peripheral oedema. She was admitted with tachypnea (respiratory rate was $32 / \mathrm{min}$ ), tachycardia (108beats/min) and blood pressurewas $125 / 76 \mathrm{mmHg}$. She was cyanosed with oxygen saturations of $89 \%$ on $60 \%$ oxygen. The jugular venous pressure was raised and pulsatile. On auscultation, there was bilateral vesicular breath sound with coarse bilateral late inspiratory crackles along with accentuated P2.Head, neck and abdominal examination was unremarkable. She had no features of rheumatological diseases.

She had a normal full blood count, renal and liver functions and electrolytes. Her CRP was only 4.7 $\mathrm{mg} / \mathrm{mL}$. Her Procalcitonin level was within the normal limits. Her sputum gram stain wasnegative, and blood and sputum cultures had no bacterial growth. Sputum direct smear was negative for acid fast bacilli and TB PCR was negative. Urine for Legionella antigen, serum for mycoplasma antibody, HIV,CMV,sputum for pneumocystis on silver stain, sputum for fungal studies and cryptococcal antigen, nasopharyngeal swabs for H1N1 all failed to identify a causative organism. Her autoimmune profile was negative for ANA titer, dsDNA, cANCA, pANCA and rheumatoid factor.Her serial arterial blood gas analysis showed a respiratory alkalosis with hypoxemia and reduced $\mathrm{PaCO} 2$.

Her chest Xray showed symmetrical reticular nodular shadows involving both lower zones. Her HRCT revealed patchy ground glass opacities and interand intra-lobular thickening with subpleural sparing involving both lungs in anterior segment of right upper lobe, apicoposterior segment of left upper lobe, 
right middle lobe, left lingular lobe and both lower lobes. Patchy areas of traction bronchiectasis were seen in rightmiddle lobe, left lingular lobe and both lower lobeswith no areas of honeycombing. Lobular air trapping was noted with no pleural effusions. The overall impressionwas subacute on chronic hypersensitivity pneumonitis.

Echocardiogram showed mild pulmonary hypertension. Pulmonary function tests and bronchoalveolar lavage could not be done during this acute clinical presentation.

She was given continuous positive airway pressure support (CPAP) in the intensive care unit (ICU). She was initially started on broad spectrum antibiotics - piperacillin tazobactam, and clarithromycin. Subsequently linezolid, clindamycin and cotrimoxazole were used. With very low procalcitonin levels and radiological evidence of subacute on chronic hypersensitivity pneumonitis she was started on intravenous methylprednisolone $750 \mathrm{mg}$ daily up to three pulses followed by high dose oral prednisolone 1 $\mathrm{mg} / \mathrm{kg} /$ day maintenance dose. Her oxygen requirements dramatically reduced within three days of glucocorticoids. The patient showed remarkable response to glucocorticoids and discharged from ICU on third day. Except for steroid induced hyperglycemia she did not have adverse effects of high dose glucocorticoids. She was also started on calcium $1200 \mathrm{mg}$ and vitamin D 800IU daily for bone protection. We suspected that this case was pigeon dustinduced hypersensitivity pneumonitis due to long duration of direct exposure at home with pigeon keeping. The pulmonary hypertensionwas thought to be secondary to chronic HP.She was discharged on oral prednisolone $30 \mathrm{mg}$ daily and planned to readmit for further evaluation in two weeks.

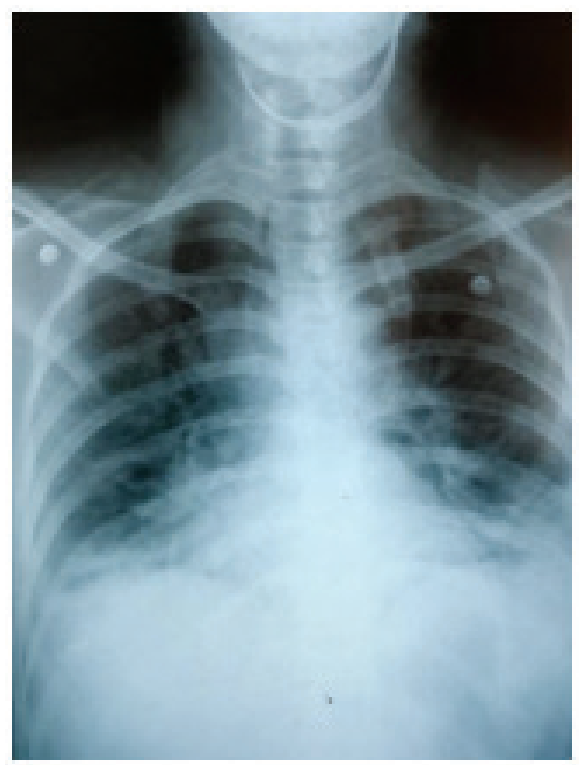

Figure 1: CXR AP of the patient on day 1 symmetrical reticular nodular shadow involving both lower zones

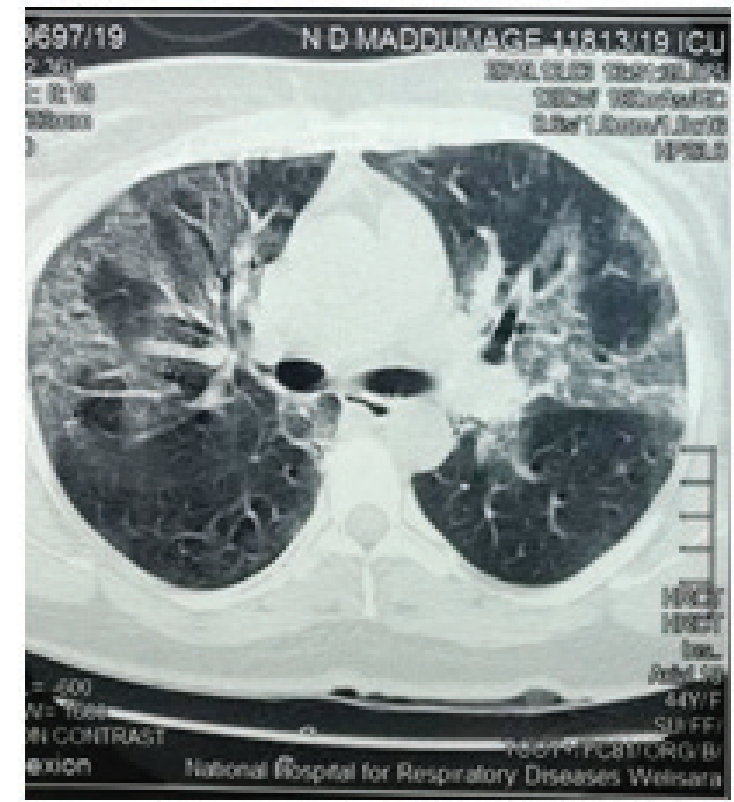

Figure 2: HRCT filmof the patient showing patchy ground glass opacities and inter- and intra-lobular thickening with subpleural sparing involving both lungs suggestive of subacute on chronic HP

\section{Discussion:}

HP can present in acute, subacute or chronic forms depending on the type, intensity and duration of exposure to the causative agent. ${ }^{1}$ The disease is histologically characterized by a triad of non-necrotizing granulomas, chronic inflammatory changes in the small airways, and diffuse interstitial infiltrates of the chronic inflammatory cells. ${ }^{2}$

Pigeon fancier's lungis caused by exposure to airborne avian antigen which provokes a hypersensitivity reaction in a susceptible host. The prevalence depends on the intensity, duration of exposure and individual host factors. 1Studies document 600021,000 cases per 100,000 persons per year for pigeon breeders in U.S but the incidence in Sri Lanka is unknown. Early diagnosis of this entity is important as it may reverse the disease and if unchecked can lead to irreversible lung damage, respiratory insufficiency and even death. 5

The diagnosis of bird fancier's lung (BFL) is of a high clinical suspicion with extensivehistory of potential hazards from occupational and animal exposure. A diagnostic criteriato aid the diagnosis has been suggested by Chan and e al.30ur patient met three major and two minor criteria.

Another clinical prediction rule as an aid to accurate diagnosis of active HP has been developed and has been demonstrated to have a maximum probability of $98 \%$ of having HP when these predictors are present. 4 Thus the requirement of BAL or lung biopsy 
would be unnecessary for the confirmation especially if HRCT is also consistent with the diagnosis 5 as in our case.

The prognosis is generally favorable with early identification, prompt removal of the offending antigen and symptomatic treatment with corticosteroids. ${ }^{1} \mathrm{~A}$ continued antigen exposure can lead to a permanent loss in lung function, irreversible pulmonary fibrosis and even premature death. ${ }^{1}$ In our patient the disease has progressed to chronic state. Steroids are also recommended for patients with severe or progressive chronic HP though its therapeutic efficacy is variable.Longterm corticosteroid therapy for the treatment of chronic HP should be considered only if objective improvements in clinical signs, pulmonary function, or radiographic abnormalities are documented. ${ }^{6} \mathrm{We}$ emphasize the importance of history in patients presenting with dyspnea in regard to potential occupational and recreational hazards because early detection and avoidance haveprovengood prognosis.

\section{References:}

1. Dickson, S. D., \& Tankersley, M. S. (2015). Fatal Hypersensitivity Pneumonitis from Exposure to in a Home Environment: A Case Report. International Archives of Allergy and Immunology, 166(2), 150-153.

2.Coleman A, Colby TV. Histologic diagnosis of extrinsic allergic alveolitis. Am J Surg Pathol 1988;12:514-8.

3. A.L. Chan, M.M. Juarez, K.O. Leslie, H.A. Ismail, T.E. AlbertsonBird fancier's lung: a state-of-the-art reviewClin. Rev. Allergy Immunol., 43 (2012 Aug), pp. $69-83$

4. M. Girard, Y. Lacasse, Y. CormierHypersensitivity pneumonitis Allergy, 64 (2009), pp. 322-334

5. V.P. Kurup, M.C. Zacharisen, J.N. FinkHypersensitivity pneumonitisIndian J. Chest Dis. Allied Sci., 48 (2000), pp. 115-128

6. Dhanasekar T, Shanmugapriya K, B. Rajagopalan, Koushik Muthuraja M. Bird Fancier s Lung: A Case Report. Journal of Evolution of Medical and Dental Sciences 2015; Vol.4, Issue 32, April 20;

Page:5592-5595, 